\title{
Imaging mass spectrometry in drug development and toxicology
}

\author{
Oskar Karlsson $^{1,2} \cdot$ Jörg Hanrieder ${ }^{3,4}$
}

Received: 1 October 2016 / Accepted: 24 November 2016 / Published online: 8 December 2016

(C) The Author(s) 2016. This article is published with open access at Springerlink.com

\begin{abstract}
During the last decades, imaging mass spectrometry has gained significant relevance in biomedical research. Recent advances in imaging mass spectrometry have paved the way for in situ studies on drug development, metabolism and toxicology. In contrast to wholebody autoradiography that images the localization of radiolabeled compounds, imaging mass spectrometry provides the possibility to simultaneously determine the discrete tissue distribution of the parent compound and its metabolites. In addition, imaging mass spectrometry features high molecular specificity and allows comprehensive, multiplexed detection and localization of hundreds of proteins, peptides and lipids directly in tissues. Toxicologists traditionally screen for adverse findings by histopathological examination. However, studies of the molecular and cellular processes underpinning toxicological and pathologic findings induced by candidate drugs or toxins are important to reach a mechanistic understanding and an effective risk assessment strategy. One of IMS strengths is the ability to directly overlay the molecular information from
\end{abstract}

Oskar Karlsson

oskar.karlsson@ki.se

1 Center for Molecular Medicine, Department of Clinical Neuroscience, Karolinska Institute, 17176 Stockholm, Sweden

2 Department of Pharmaceutical Biosciences, Drug Safety and Toxicology, Uppsala University, 75124 Uppsala, Sweden

3 Department of Psychiatry and Neurochemistry, Sahlgrenska Academy at the University of Gothenburg, Mölndal Hospital, House V, 43180 Mölndal, Sweden

4 Department of Molecular Neuroscience, UCL Institute of Neurology, University College London, Queen Square, London WC1N, UK the mass spectrometric analysis with the tissue section and allow correlative comparisons of molecular and histologic information. Imaging mass spectrometry could therefore be a powerful tool for omics profiling of pharmacological/ toxicological effects of drug candidates and toxicants in discrete tissue regions. The aim of the present review is to provide an overview of imaging mass spectrometry, with particular focus on MALDI imaging mass spectrometry, and its use in drug development and toxicology in general.

Keywords MALDI IMS · SIMS · BMAA · Toxins ·

Neurotoxicology $\cdot$ Preclinical $\cdot$ Biologics $\cdot$ Small-molecule drugs $\cdot 6-O H D A \cdot$ L-DOPA

\section{Introduction}

Drug development requires detailed information of absorption, distribution, metabolism, excretion and toxicity (ADMET) properties of the novel drug candidates. Most drugs or metabolites are not uniformly distributed, and characterizing the tissue distribution of the drug candidate is key to understand other parts of drug development. Therapeutic agents need to be well distributed to their intended target site to have the desired pharmacological effect (Lanao and Fraile 2005; Mouton et al. 2008). Moreover, accumulation of parent drug or metabolites in unexpected tissues may lead to untargeted secondary pharmacology and toxicity (Castellino et al. 2011; Pellegatti and Pagliarusco 2011). Studies of compound distribution are not only important for preclinical safety assessment but also for mechanistic studies and risk assessment of environmental contaminants. Today most of our knowledge of drug or toxicant tissue localization is derived from whole-body autoradiography or homogenate LC-MS analysis (Castellino 
et al. 2011; McEwen et al. 2014). However, during the last decades imaging mass spectrometry (IMS) techniques that use various ionization modes such as desorption electrospray ionization (DESI), matrix-assisted laser desorption/ ionization (MALDI), nanoparticle laser desorption/ionization (nano-PALDI) and secondary ion mass spectrometry (SIMS) have emerged as powerful alternatives for chemical imaging in situ (Hanrieder et al. 2015; Sugiura and Setou 2010; Waki et al. 2015).

Different imaging MS technologies have various strengths and limitations particularly with respect to spatial resolution and molecular information (Hanrieder et al. 2015). In contrast to whole-body autoradiography that images the localization of radiolabeled compounds, IMS provides the opportunity to simultaneously determine the discrete tissue distribution of the parent compound and its metabolites (Khatib-Shahidi et al. 2006; Solon et al. 2010; Stoeckli et al. 2007; Sugiura and Setou 2010). In addition, IMS features high molecular specificity and allows comprehensive, multiplexed detection and localization of hundreds of proteins, peptides and lipids in biological tissue samples (Cornett et al. 2007; McDonnell and Heeren 2007; Schwamborn and Caprioli 2010). IMS could therefore be used for omics profiling of pharmacological/toxicological effects of drug candidates and toxicants in discrete tissue regions. The increased relevance and popularity of IMS in biomedical and basic molecular biology research is reflected in the steady increase in IMS publications (Fig. 1). However, only few reports on using this technology for monitoring drugs, drug metabolites as well as drug safety and toxicological studies are available yet (Fig. 1), highlighting the challenges of employing IMS for such analyses due to e.g., matrix interference, isobaric interferences and sample throughput. The aim of the present review is to provide an overview of IMS, with particular focus on MALDI IMS, and its use in drug development and toxicology in general.

\section{Imaging mass spectrometry: principles and modalities}

In IMS, the analyte localization is obtained by sequential acquisition of mass spectra from a predefined pixel array over a biological sample, i.e., tissue section or cell preparation (Fig. 2a, b). A major challenge in IMS is desorption and ionization of target species for subsequent MS analysis. Various imaging MS modalities have been developed over the last decades with MALDI, SIMS and DESI-based IMS being the most prominent. In SIMS, analytes are desorbed and ionized through impacting the sample with a focused ion beam, leading to formation and ejection of secondary ions (Colliver et al. 1997). In MALDI, a crystalline UV-absorbing matrix is used for enhancing analyte desorption and ionization through charge transfer in the gas phase (Hillenkamp et al. 1991; Karas and Hillenkamp 1988). In DESI, an electrospray is used for desorbing molecular species and generate ions, followed by transfer to the mass analyzer (Laskin et al. 2012; Takats et al. 2004). All ionization principles and IMS modalities have strengths and limitations, which have to be addressed by appropriate experimental design and by using the right technique for the right purpose.

In IMS, single ion images are generated by plotting the intensity pattern of a distinct molecular species over the analyzed tissue array (Fig. 2c). The spot-to-spot distance of the tissue array defines the pixel size and spatial resolution of the IMS analysis, respectively. The spatial resolution is mainly hampered by the probe-ion beam in SIMS, laser in MALDI and electrospray in DESI-but also lateral diffusion during sample preparation (MALDI) and analysis (DESI) has a major impact. In SIMS, spatial resolution below $1 \mu \mathrm{m}$ and down to $30 \mathrm{~nm}$ is possible, but this is accompanied with compromises of the mass resolution and eventually the sensitivity (Benabdellah et al. 2010). Likewise, high spatial resolution MALDI IMS
Fig. 1 Publication statistics. Annual numbers of published articles concerning imaging mass spectrometry and its application to drug discovery and toxicology. Data retrieved from PubMed

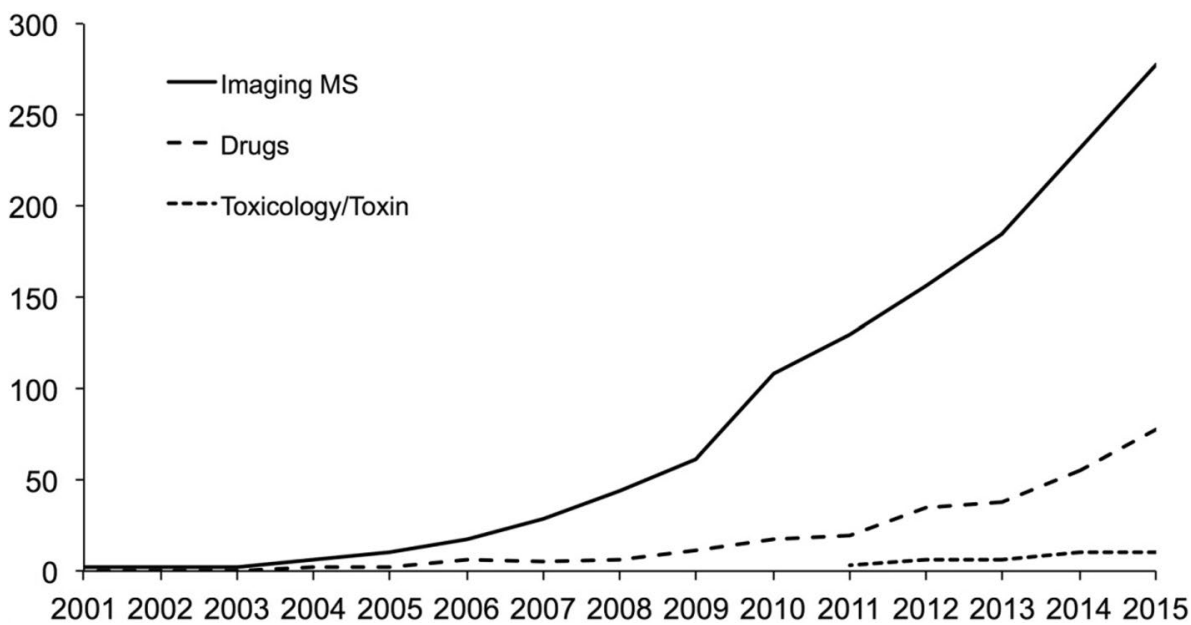




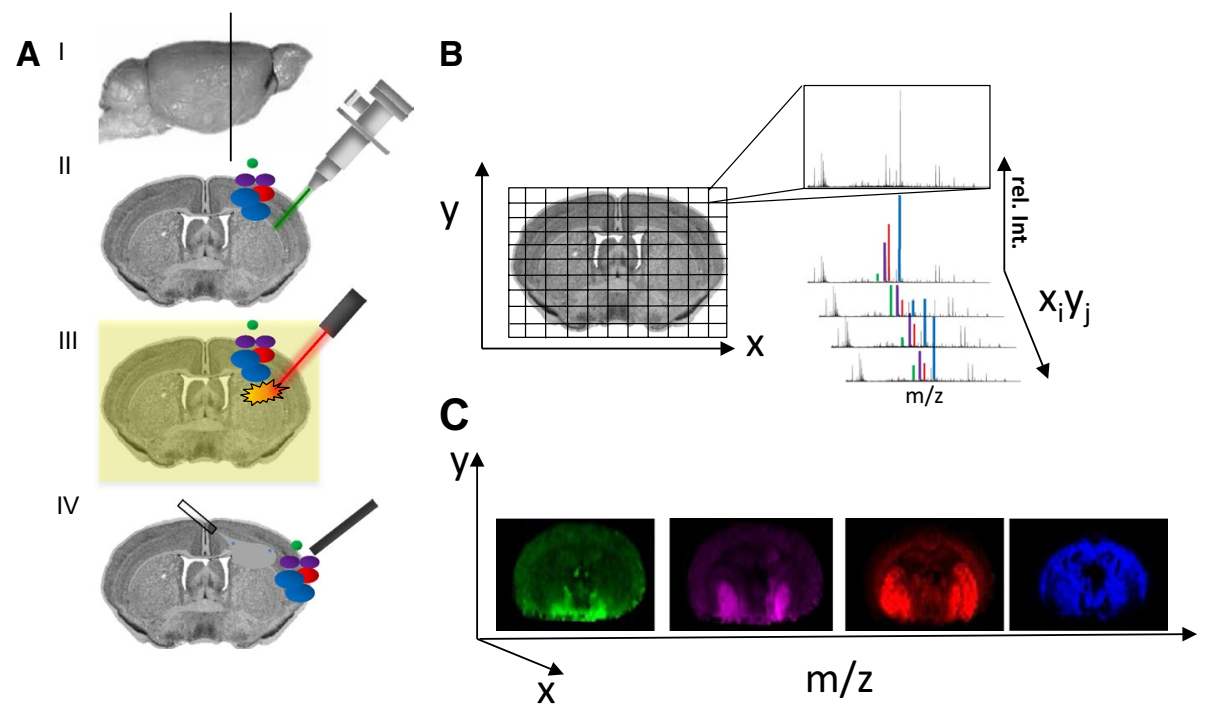

Fig. 2 Principle of imaging mass spectrometry. a $I$ Tissue sections are collected and mounted on a target for imaging mass spectrometry. II For SIMS IMS, tissue sections are probed with an ion beam, generating low molecular weight secondary ions $(\mathrm{m} / z>1000 \mathrm{Da})$. III In contrast, MALDI IMS requires pre-coating with matrix (indicated in yellow) before systematic scanning with a laser probe. MALDIbased ionization generates larger intact molecular species, includ-

experiments of up to $1-10 \mu \mathrm{m}$ are limited by the sensitivity of the method to the respective compounds of interest (Benabdellah et al. 2010). The spatial resolution may also be limited due to practical reasons including acquisition time bias and data size as there is a complete MS spectrum for each pixel in the acquired image. Analysis of complex imaging data can be performed using multivariate statistical analysis (MVSA) tools, such as principal component analysis (PCA) and maximum autocorrelation factor analysis (MAF). Multiple image analysis strategies have been reported for SIMS (Graham and Castner 2012; Henderson et al. 2009; Tyler et al. 2007) and MALDI IMS data (Deininger et al. 2008). This approach allows unbiased segmentation of a biological sample into distinct regions of interest by detecting variances and correlations in the multivariate data that are encompassed in the MVSA factors (PC or $\mathrm{MAF}$ ). From the corresponding scores and loadings, the variables that are contributing the most (i.e., mass peak values) to these variances can be deduced revealing histology associated chemical changes.

\section{MALDI imaging mass spectrometry}

The introduction of the soft ionization technique MALDI for mass spectrometry had a significant impact on the field of biological and biomedical sciences. This technology facilitates rapid and specific characterization of intact large ing peptides and proteins. IV In DESI IMS, a focused electro spray ionization probe is used to sample the tissue. The desorbed ions are collected with a co-aligned capillary that is connected to the mass spectrometer. b One mass spectrum is acquired for every $x_{\mathrm{i}}, y_{\mathrm{j}}$ coordinate of the scanned tissue section. c Single ion images are generated by mapping the intensity of an individual ion signal $(\mathrm{m} / \mathrm{z}$; rel.Int) over the whole tissue array

biomolecular species, including proteins and peptides, which fueled the development of proteomics (Karas and Hillenkamp 1988). The technique is based on laser irradiation induced desorption and ionization of intact molecular species with the help of a crystalline UV-absorbing matrix (Fig. 2a). The generated ions are typically characterized with a time-of-flight (TOF) mass analyzer, but other mass analyzers such as Fourier transform ion cyclotron resonance (FTICR) or orbitrap can also be used. MALDI MS is characterized by its great mass range, sensitivity, high mass resolution and mass accuracy as well as its robustness, acquisition speed and insensitivity to sample impurities. MALDI IMS was introduced in 1997 by Caprioli et al. (1997). The technique is particularly well suited for medium to large biomolecules including glycolipids, neuropeptides and proteins. Spatial resolution in MALDI IMS is essentially dependent on three factors-the laser beam focus, the matrix crystal size and lateral diffusion of analyte molecules resulting during sample preparation. Efforts to push the spatial resolution with respect to the probe have been reported (Altelaar et al. 2007; Jurchen et al. 2005; Spengler and Hubert 2002). However, in large-scale experiments a practical spatial resolution of 10-20 $\mu \mathrm{m}$ may be achieved, but typically a $50-300 \mu \mathrm{m}$ resolution is used. This can be compared to whole-body autoradiography, which has a spatial resolution around $5 \mu \mathrm{m}$ when using film, and 50-100 $\mu \mathrm{m}$ when using phosphor detection methods for quantification (McEwen et al. 2014). 


\section{Sample preparation}

For MALDI IMS, the sample preparation significantly impacts the final data quality, particularly with respect to signal intensity, reproducibility, as well as spatial resolution. The sample preparation starts with tissue collection and storage, continues with tissue sectioning, sample wash and final matrix application. Each of these steps is critical for the final data quality. In particular, matrix application is a key aspect in the MALDI IMS experimental workflow and should be tailored according to numerous factors, including tissue origin and molecular target.

\section{Tissue collection}

For tissue imaging, adequate tissue collection is critical for data quality. Commonly used perfusion and fixation strategies are generally not compatible with mass spectrometry, due to interference of the polymeric fixation agents such as paraffin. Solutions to overcome this problem, including in situ trypsinization following paraffin removal or alternatively use of a reactive matrix 2,4-dinitrophenylhydrazine, have been suggested (Lemaire et al. 2007; Ly et al. 2016). Another elegant workaround was recently presented to delineate spatial protein N-glycosylation patterns (Gustafsson et al. 2015). These approaches, however, do not permit analysis of endogenous lipids, peptides and intact proteins due to washing steps with organic solutions (lipids, endogenous peptides) and enzymatic degradation (proteins). Fresh frozen tissues are therefore the most suitable and commonly used samples for IMS of all target species including low molecular weight compounds such as drugs, metabolites, and toxicants. A standardized protocol for quick and diligent tissue retrieval and snap freezing is essential, since postmortem delays by as little as 3-min result in severe degradation of proteins and peptides, truncating the correct representation of endogenous species and drug metabolites (Goodwin et al. 2008). Fresh frozen tissue samples are stored at $-80{ }^{\circ} \mathrm{C}$ until preparation for IMS by cryosectioning. Tissue sections are collected with a cryostat microtome at -17 to $-20{ }^{\circ} \mathrm{C}$ and thaw mounted onto conductive sample surfaces such as glass slides covered with indium tin oxide (ITO) or silicon wafers. The collected sections are dried immediately before storage at $-80{ }^{\circ} \mathrm{C}$ in order to prevent damage by water condensation during freezing (Hanrieder et al. 2012).

\section{Tissue washing}

The choice of appropriate washing protocols is also a critical step in sample preparation. Lipid species typically do not require any advanced washing steps, whereas drugs, neuropeptides and proteins require optimized washing protocols for signal enhancement. These involve $\mathrm{pH}$ sensitive cleanup as well as organic solvents for precipitation of peptides and proteins while washing off remaining lipids that potentially interfere with the analyte signals prior to matrix application (Hanrieder et al. 2012; Shariatgorji et al. 2012a). Several washing protocols have been evaluated for enhancing protein signal in MALDI imaging. Stepwise washing with gradient alcohol has been found to give the most significant improvement in protein signal quality (Martin-Lorenzo et al. 2014; Seeley et al. 2008).

\section{Matrix application}

Different matrices are well suited for different compounds and the choice depends largely on the targeted analyte. A suitable MALDI matrix should be stable and enable ionization of the analyte without producing matrix-derived peaks in the $\mathrm{m} / \mathrm{z}$ region of interest. Common matrices include $\alpha$-cyano-4-hydroxycinnamic acid (CHCA), 2,5-dihydroxybenzoic acid (DHB), sinapinic acid (SA) and 9-aminoacridine (9-AA). The matrix should be applied in a standardized way that allows adequate extraction of the analyte while minimizing its delocalization. Several manual and automatic techniques have been used for matrix application including spotting, spray coating and sublimation, each with its own strengths and limitations with respect to simplicity, cost, resulting signal intensity, spatial resolution and signal reproducibility (Hanrieder et al. 2015).

\section{MALDI-based intact protein imaging}

MALDI IMS is well suited for in situ protein analysis with retained spatial information. Although IMS is a powerful technique, there is still a need for complementary validation strategies to rule out false-positive findings as a result of experimental factors such as suppression effects and peak identification. Common validation approaches include immunohistochemistry, LC-MS/MS for proteomics and peptidomics in tissue extracts as well as in situ fragmentation (MS/MS) for top-down protein and peptide identification. A comprehensive list of identified neuropeptides and proteins observed in MALDI IMS experiments has previously been published (Hanrieder et al. 2015).

The application of MALDI IMS to projects of biological and clinical significance has increased since the introduction of the technique (Caprioli et al. 1997). However, many IMS publications are still of methodological character and are based on tissue sections from one or a few subjects. Some biological relevant experiments retrieve statistical information on different treatment groups directly from IMS data (Hanrieder et al. 2011, 2013; Jones et al. 2011; Karlsson et al. 2012, 2014; Kriegsmann et al. 2012; Ljungdahl et al. 2011; Meistermann et al. 2006; Onishi 
et al. 2013; Oppenheimer et al. 2010; Willems et al. 2010). Other studies rely on histology-directed profiling experiments to generate data for statistical analysis, followed by a single IMS experiment of one or two sections to elucidate the localization of interesting peptides and proteins (Burnum et al. 2009; Norris et al. 2007; Stauber et al. 2008; Uys et al. 2010; Yanagisawa et al. 2003). This may be a sensible rationale when the regions of interest are known prior to the experiment. By contrast, IMS can be used as an exploratory tool to localize affected organs or tissue regions (Hanrieder et al. 2013; Karlsson et al. 2012, 2014).

\section{MALDI imaging mass spectrometry for localization and quantification of drugs and toxicants}

The major advantage of MALDI IMS for distribution studies is the possibility to simultaneously detect the parent molecule and its metabolites (Nilsson et al. 2015). However, detection of these small molecules is hampered by interference of matrix cluster ions. This limitation can be overcome by matrix-free laser desorption/ionization (LDI) approaches as well as other strategies including e.g., use of a stable isotope modified matrix, derivatization or MS/MS methodologies (Nilsson et al. 2010; Northen et al. 2007; Prentice et al. 2016; Shariatgorji et al. 2012b, 2015). The first experiment designed to study drug distribution using MALDI IMS in a whole-body mouse section was published in 2005 by Rohner et al. (2005). This approach was then extended for combined studies on drug/metabolite localization and mapping of endogenous biomarkers (Khatib-Shahidi et al. 2006). Quantification of target molecules by MALDI IMS is challenging but important for future integration of IMS in toxicological studies/evaluation. The sample preparation, in particular the matrix application, is essential for the data quality and reproducibility (see above). Moreover, when performing quantification of a target molecule in different organs or tissue regions ion suppression-mainly caused by different lipid and salt concentrations-could be a problem. To reduce the variability of IMS data, different strategies have been used to compensate for matrix crystallization effects and ion suppression during data acquisition. Approaches used include normalizing the spectra against the total ion count (TIC), other calculated normalization factors, or the signal intensity of an internal standard applied to the tissue section (Hamm et al. 2012; Hochart et al. 2014; Kallback et al. 2012; Stoeckli et al. 2007). The majority of published research concerns the quantification of smallmolecule drugs (Chumbley et al. 2016; Groseclose and Castellino 2013; Koeniger et al. 2011; Nilsson et al. 2010). For example, the antibiotics rifampicin was quantified in pooled human plasma as well as in liver tissue from an animal dosed in vivo using a TOF/TOF instrumentation and a MS/ MS approach with rifapentine as an internal standard. This enables multiple fragmentation events to be performed in a single laser shot, allowing the intensity of the analyte to be referenced to the intensity of the internal standard in each laser shot while maintaining the benefits of MS/MS (Prentice et al. 2016). Today, an increasing number of pharmaceutical companies are trying to develop therapeutic peptides or proteins including antibodies. A recent distribution study of the somatostatin analog octreotide shows that this synthetic octapeptide was clearly visualized and quantified by MALDI IMS (Takai et al. 2013). This suggests that IMS could be a suitable technique for ADMET studies not only for small-molecule drugs but also therapeutic peptides and other biological drugs.

The MALDI IMS methods developed for distribution and quantification of pharmaceutical drugs in tissues can also be applied for toxicants. For example, quantitative brain imaging of the environmental neurotoxin $\beta$ - $N$-methylamino-L-alanine (BMAA) - suggested to be involved in neurodegenerative disorders (Cox et al. 2016; Spencer et al. 1987)—was performed after derivatization with 2,4-diphenyl-pyranylium (DPP) by relating the signals for BMAA in dosed rats a calibration curve constructed by spotting known concentrations of BMAA on a control tissue section (Shariatgorji et al. 2015). Moreover, it has been demonstrated that IMS can be used for quantification of the organochloride pesticide chlorodecone in the mouse liver (Lagarrigue et al. 2014). The method combines normalization by an internal standard in the matrix solution and the correlation with an orthogonal technique to achieve in situ absolute quantification (Lagarrigue et al. 2014). In addition, MALDI IMS has been used to study the brain distribution of the neurotoxic metabolite 1-methyl-4-phenylpyridinium (MPP+) after administration of 1-methyl-4-phenyl-1,2,3,6tetrahydropyridine (MPTP) in mice (Kadar et al. 2014).

\section{Imaging mass spectrometry in mechanistic toxicology}

Toxicologists traditionally screen for adverse findings by histopathological examination of tissues and monitoring selected biomarkers in urine or plasma. However, studies of the molecular and cellular processes underpinning toxicological and pathologic findings induced by drug candidates or toxicants are important to reach a mechanistic understanding and an effective risk assessment strategy (Ahuja and Sharma 2014). One of the strengths of IMS is the ability to directly overlay the molecular information from the mass spectrometric analysis with the tissue section and allow correlative visual comparisons of molecular and histologic information. 


\section{Organ toxicology}

Recent studies demonstrate the emerging role of IMS in the discovery of toxicity biomarkers and in obtaining mechanistic insights concerning toxicological mechanisms. For example, dabrafenib (DAB) is a drug approved for the treatment of specific tumors in adults. Preclinical studiesconducted to support the use of DAB in treatment of pediatric tumors-have shown drug-induced pathological effects on the kidney of juvenile rats. Tubular deposits were considered to be responsible for the renal pathogenesis due to effects of obstructive nephropathy (Groseclose et al. 2015). IMS analysis of juvenile rat kidneys contributed to a more thorough mechanistic understanding of the nephrotoxicity compared to LC-MS homogenate data alone, by characterizing tissue localization of DAB and its metabolites as well as determining the chemical composition of the renal deposits (Groseclose et al. 2015). The IMS results revealed that the tubular deposits consisted primarily of calcium phosphate and did not contain any drug-related material. This data allow a more complete risk assessment for pediatric treatment with DAB (Groseclose et al. 2015). MALDI IMS combined with data from manual tissue dissection analyzed by LC-MS/MS, and NMR has also been used to successfully identify the composition of renal deposits induced by two potential microsomal prostaglandin E synthase 1 (mPGES-1) inhibitors (Nilsson et al. 2012). The renal deposits consisted of their common metabolite bisulfonamide and an un-targeted analysis revealed molecular changes in the kidney that were specifically associated with the area of the tissue defined as pathologically damaged (Nilsson et al. 2012). Similarly, in a toxicological study of a drug candidate, MALDI IMS experiments revealed accumulation of the active drug in multiple organs where microcrystalline deposits were observed following administration of the pro-drug (Drexler et al. 2007). In a more recent study, MALDI IMS and DESI IMS were used to determine the composition of drug-induced crystal-like structures. PCA analysis of the MALDI IMS data revealed together with the MS/MS results that the crystal-like structures in the kidney of dosed rabbits are mainly composed of metabolites originating from demethylation and/or oxidation of the drug (Bruinen et al. 2016). In addition, the technique has been used to identify a transthyretin fragment (Ser28-Gln146) as a potential toxicity biomarker for the well-known nephrotoxicant gentamicin (Meistermann et al. 2006).

\section{Neurotoxicology}

Many pathological processes are characterized not by large changes in the transcriptome or proteome, but by discrete changes in a subset of cells or specific loci. This may be particularly true for many pathophysiological processes in the brain (Hanrieder et al. 2015). The brain is highly organized in topographic maps and functionally related circuitries that might not be easily recognized or isolated for traditional proteomic analysis (Hanrieder et al. 2015; Karlsson et al. 2014). Hence, IMS may be particularly useful for mechanistic neurotoxicology. This is well illustrated by studies of the environmental neurotoxin BMAA and neurotoxin-based animal models of Parkinson's disease (PD).

\section{MALDI imaging mass spectrometry as a molecular exploratory tool: long-term effects of neonatal exposure to BMAA}

BMAA is transferred via breast milk to nursed offspring (Andersson et al. 2013, 2016) and demonstrated to induce long-term cognitive impairments after neonatal exposures of rats (Karlsson et al. 2009a, b, 2011). MALDI IMS has been used as an exploratory tool in combination with histopathology to identify brain regions affected by the neonatal BMAA administration (Fig. 3a). An initial screening of sagittal sections from the left hemispheres of rat brains collected 6 months after toxin exposure revealed BMAAinduced protein changes in several brain regions including the striatum and hippocampus (Karlsson et al. 2014). These regions were selected for full-scale experiments using coronal cryosections from the right hemispheres of all 21 animals (Karlsson et al. 2012, 2014). The striatum was divided into caudate putamen $(\mathrm{CPu})$ and nucleus accumbens (NAc) and the regional analysis revealed 10 and 18 significantly altered protein peaks in the $\mathrm{CPu}$ and the NAc of both high- and low-dose groups compared to controls. Decreased levels of myelin basic protein (MBP) were demonstrated (Fig. 3b, c) and indicate that developmental exposure to BMAA could induce structural effects on axonal growth and/or directly on the proliferation of oligodendrocytes and myelination, which might be important for the observed long-term cognitive impairments (Karlsson et al. 2014). The IMS analysis of hippocampus revealed a decreased expression of proteins involved in energy metabolism and intracellular signaling in the adult hippocampus of both dose groups (Karlsson et al. 2012). In addition, the high BMAA dose induced changes in the expression of $\mathrm{S} 100 \beta$, histones, calcium- and calmodulinbinding proteins and guanine nucleotide-binding proteins. Interestingly, at this dose also severe lesions in the adult hippocampal CA1 region including neuronal degeneration, cell loss, calcium deposits and astrogliosis were observed (Karlsson et al. 2012). Ultrastructural examination by transmission electron microscopy revealed intracellular deposition of abundant bundles of closely packed parallel fibrils in neurons, axons and astrocytes of the CA1. SIMS IMS and laser capture microdissection 

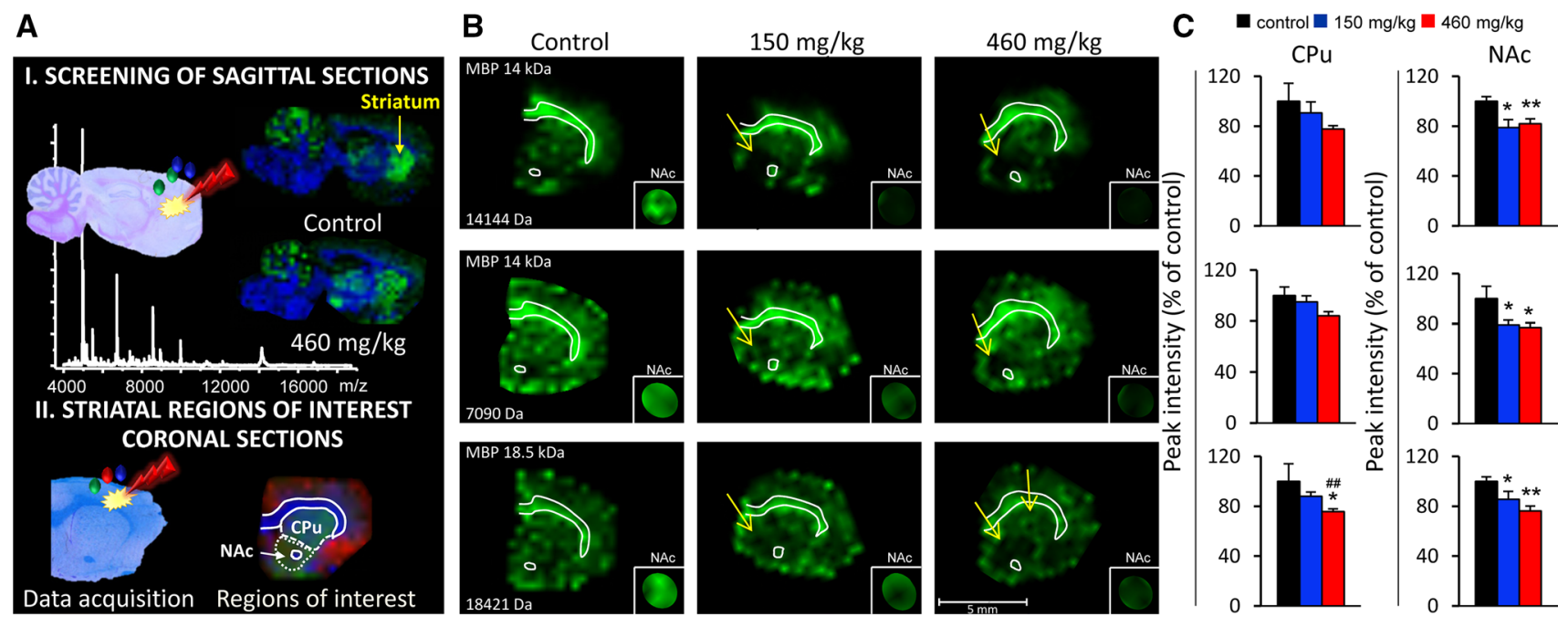

Fig. 3 MALDI imaging mass spectrometry as a molecular exploratory tool in toxicology. Neonatal rats were treated with BMAA (150 and $460 \mathrm{mg} / \mathrm{kg}$ ) or vehicle and their brains processed for MALDI IMS at adult age. a $I$ To identify affected brain regions, an initial screening of sagittal brain sections from the left hemisphere of animals in the high treatment group and control group is performed. BMAA-induced changes in peak intensities are revealed in several brain areas. II Striatum is selected for further studies in a full-scale experiment and coronal striatal cryosections are obtained from the right hemisphere of all 21 animals. For statistical analysis, distinct regions of interest are determined. Histology as well as ion distribution images of several proteins are used to define striatum, divided into caudate putamen $(\mathrm{CPu})$ and nucleus accumbens (NAc). b The analysis revealed a dose-dependent decrease in MBP levels in the $\mathrm{CPu}$ and NAc of adult rats neonatally treated with BMAA. The $14 \mathrm{kDa}$ (both singly and doubly charged) and $18.5 \mathrm{kDa}$ MBP isomers are shown in green. The arrows indicate the regional location of reduced protein expression. The small insertions are magnifications $(\times 1.5)$ of NAc with the protein of interest displayed at higher color intensity and surrounding signals blocked by a mask. $\mathbf{c}$ The bar histograms show the peak intensity (\% of control \pm SEM) for the selected proteins in $\mathrm{CPu}$ and NAc. ${ }^{*} p<0.05,{ }^{* *} p<0.01$ compared with vehicle control animals, ${ }^{\# \#} p<0.01$ compared with $150 \mathrm{mg} /$ $\mathrm{kg}$ (Kruskal-Wallis test and Mann-Whitney $U$ test). Control $n=6$; BMAA $150 \mathrm{mg} / \mathrm{kg} n=8 ; 460 \mathrm{mg} / \mathrm{kg} n=7$. Adapted from: Karlsson et al. Mol Cell Proteomics. 2014; 13(1):93-104 followed by LC-MS/MS showed enrichment of specific phospholipids and proteins in this brain region (Hanrieder et al. 2014a, b; Karlsson et al. 2015a, b). These molecular studies of BMAA-induced effects further illustrate the usefulness of combining IMS with other experimental techniques to identify target regions and investigate toxicological events.

\section{Imaging of neurotoxin-based animal models mimicking Parkinson's disease and L-DOPA-induced dyskinesia}

Understanding the molecular pathways that underlie neurodegenerative diseases is an ongoing challenge in which MALDI IMS can prove to be helpful. Several animal models of neurodegenerative disease, particularly PD, are based on injection of neurotoxins such as MPTP or 6-OHDA for selective degeneration of nerve cells mimicking neurodegeneration and impairment of distinct neuronal circuits (Karlsson and Lindquist 2016; Ungerstedt 1968). MALDI IMS has been used to validate the reduction in the calmodulin-binding protein PEP-19 and determine its brain distribution in the MPTP mouse model of PD (Skold et al. 2006). In addition, in situ mass spectrometry based profiling has been used to delineate spatial molecular changes in the striato-nigral circuit following 6-OHDA injection of mice (Pierson et al. 2004). Similarly, endogenous peptide levels were outlined in rat brain using MALDI IMS in a 6-OHDA rat model of PD (Hanrieder et al. 2011, 2012; Ljungdahl et al. 2011, 2013). Detection and quantification of endogenous neuropeptides in situ is challenging as commonly used antibody-based techniques are hampered by several factors including throughput, quantification and specificity. Antibody-based techniques require a priori knowledge of the target species and are limited in terms of throughput as it only allows detection of few species at the same time. Most importantly, immunohistochemistry of neuropeptides is significantly hampered by antibody specificity, which is particularly relevant for opioid peptides. These peptide species differ in only a few C-terminal amino acids, which compromise the reliability of immunohistochemistry results significantly. In addition, immunohistochemistry provides only semi-quantitative information. In contrast, neuropeptidomics approaches using LC-MS on tissue extracts-despite being a powerful approach for endogenous peptide characterization (Karlsson et al. 2013; Nilsson et al. 2009)—are limited in that the dissection and tissue extraction result in loss of spatial information. This highlights the need for a molecular imaging technique to 

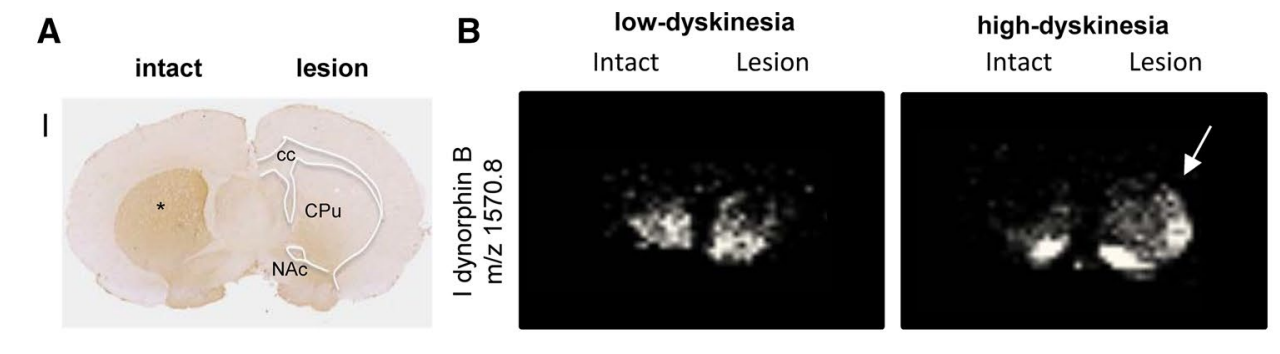

C
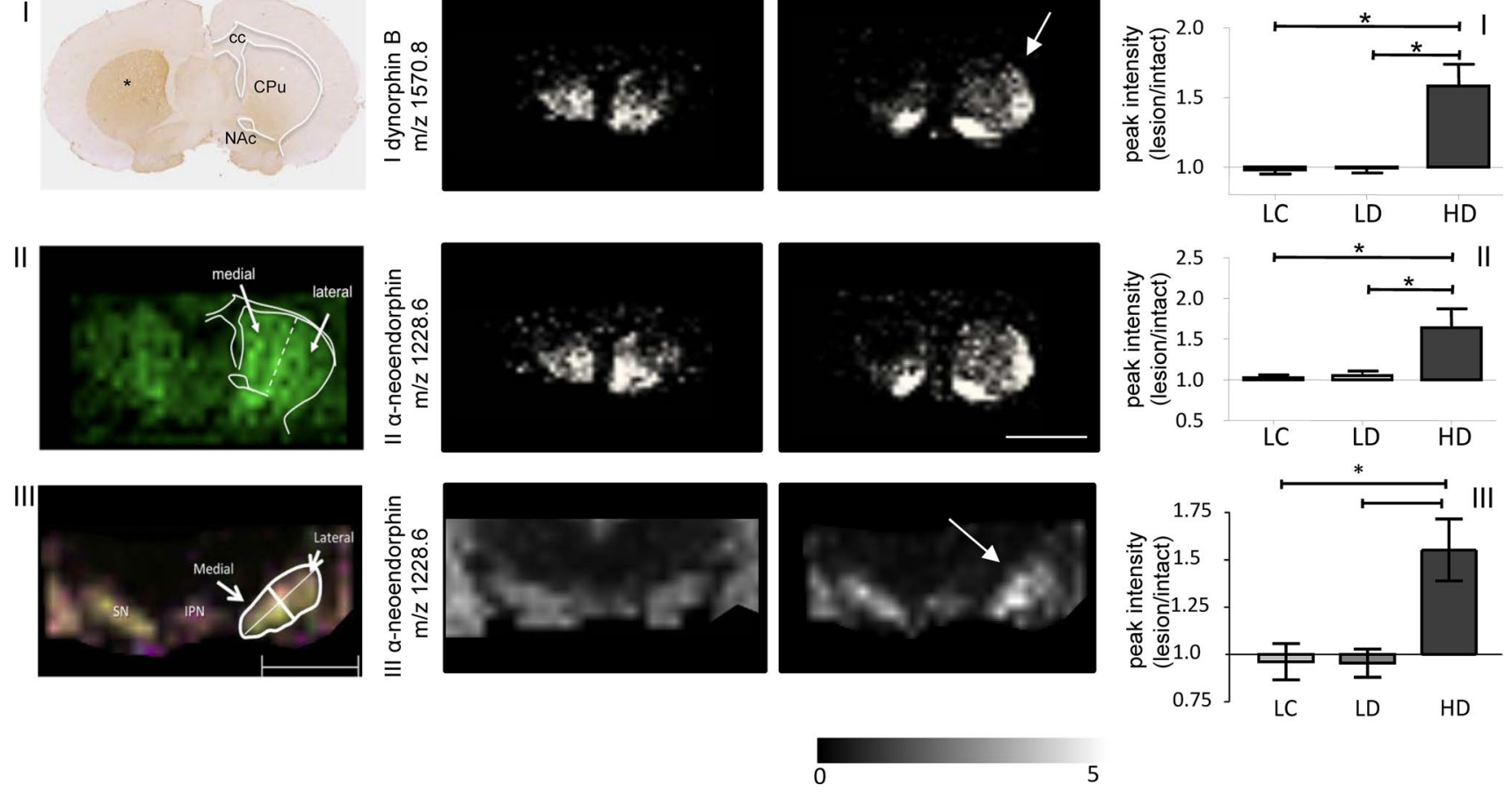

Fig. 4 MALDI imaging mass spectrometry analysis of neuropeptides in L-DOPA-induced dyskinesia. a $I$ Unilateral 6-OHDA injection leads to dopamine depletion (illustrated by tyrosine hydroxylase immunostaining*). L-DOPA therapy results in two distinct groups with low and high dyskinesia. II Striatal and III substantia nigra sections are analyzed by MALDI IMS and regions of interest (ROI) are assigned for spectral data extraction. b Dynorphin peptides, $I$ dynorphin B and II alpha neoendorphin were significantly increased in the

comprehensively delineate neuropeptide regulations in situ something that can be achieved by using IMS.

L-DOPA-induced dyskinesia (LID) is a form of dyskinesia that occurs in up to $80 \%$ of all PD patients after 5-10 years of L-DOPA treatment (Ahlskog and Muenter 2001). MALDI IMS has been employed to characterize spatial regulations of dynorphin opioid peptides in LID using the PD model based on unilateral 6-OHDA lesions in rat brain (Fig. 4) (Hanrieder et al. 2011; Ljungdahl et al. 2011). In this model, animals develop PD pathology only on one side and the other side can serve as an internal control (Ungerstedt 1968). This offers an elegant solution to avoid the effects of intra-sample group variation, which is particularly relevant IMS and mass spectrometry in general as this approach accounts for variation induced by suppression effects. Following 6-OHDA lesion and L-DOPA treatment, MALDI imaging revealed that dynorphin B and alpha neoendorphin were significantly elevated in the dorsal lateral striatum in the high dyskinetic group but not for low dyskinetic animals (Fig. 4b I-II) (Hanrieder et al. 2011). In addition, both dynorphin species correlated

dorsolateral striatum of high (HD) compared to low dyskinetic (LD) animals and lesion controls (LC). III Similarly, endogenous dynorphin peptides (e.g., alpha neoendorphin) were increased in the substantia nigra reticulata on the parkinsonian side following L-DOPA treatment. c The results are verified by spectral analysis and nonparametric ANOVA and post hoc statistics. Scalebar $2 \mathrm{~mm}$. Adapted from: Ljungdahl et al. PLoS One. 2011; 6(9) and Hanrieder et al. Mol Cell Proteomics. 2011; 10(10)

positively with LID severity (Fig. 4c I-II). Similarly, the dynorphin peptides were elevated in the substantia nigra that constitutes the main output structure of the striatal projections in the direct pathway of motor control (Fig. 4a-c III) (Ljungdahl et al. 2011).

\section{Concluding remarks}

In drug development, information about the in vivo distribution of a drug candidate after its administration is essential as it facilitates the understanding of the mechanisms underlying the efficacy or toxicity of the drug. Despite recent advances in IMS methodologies including MALDI IMS, whole-body autoradiography based on the method of Ullberg published more than 60 years ago (Ullberg 1954, 1977) still remains the gold standard for determining tissue distribution of drugs (McEwen and Henson 2015; McEwen et al. 2014). One of the strengths of IMS, compared to whole-body autoradiography, is the opportunity to simultaneously determine the discrete tissue distribution of the parent compound its 
metabolites as well as endogenous molecules. This raises the exiting possibility of performing experiments that monitor both distribution and pharmacological/toxicological mechanisms at the same time. However, IMS of small molecules is limited by endogenous and matrix-related overlapping (isobaric) peaks. The instrument must therefore be able to differentiate the target compounds from the background noise. This is usually achieved by performing the experiments in MS/MS mode, which impairs a simultaneously and untargeted analysis of multiple analytes. Future developments in instrument performance and methodologies are needed to simplify the experimental procedure, provide reliable quantitative data and to streamline on tissue omics profiling. At present, IMS could be considered as a complementary method that may give valuable information during preclinical studies of drug candidates as well as in toxicological research. As the work with technical improvements is continuously ongoing, IMS could be expected to become a standard method in drug development and toxicology (Fisher et al. 2016; Ogrinc Potocnik et al. 2015; Prentice et al. 2016).

Open Access This article is distributed under the terms of the Creative Commons Attribution 4.0 International License (http://creativecommons.org/licenses/by/4.0/), which permits unrestricted use, distribution, and reproduction in any medium, provided you give appropriate credit to the original author(s) and the source, provide a link to the Creative Commons license, and indicate if changes were made.

\section{References}

Ahlskog JE, Muenter MD (2001) Frequency of levodopa-related dyskinesias and motor fluctuations as estimated from the cumulative literature. Mov Disord 16(3):448-458

Ahuja V, Sharma S (2014) Drug safety testing paradigm, current progress and future challenges: an overview. J Appl Toxicol 34(6):576-594. doi:10.1002/jat.2935

Altelaar AFM, Taban IM, McDonnell LA et al (2007) High-resolution MALDI imaging mass spectrometry allows localization of peptide distributions at cellular length scales in pituitary tissue sections. Int J Mass Spectrom 260(2-3):203-211. doi:10.1016/j. ijms.2006.09.028

Andersson M, Karlsson O, Bergstrom U, Brittebo EB, Brandt I (2013) Maternal transfer of the cyanobacterial neurotoxin beta- $N$-Methylamino-L-Alanine (BMAA) via milk to suckling offspring. PLoS ONE 8(10):e78133. doi:10.1371/journal.pone.0078133

Andersson M, Karlsson O, Banack SA, Brandt I (2016) Transfer of developmental neurotoxin beta- $N$-methylamino-L-alanine (BMAA) via milk to nursed offspring: studies by mass spectrometry and image analysis. Toxicol Lett 258:108-114. doi:10.1016/j.toxlet.2016.06.015

Benabdellah F, Seyer A, Quinton L, Touboul D, Brunelle A, Laprevote O (2010) Mass spectrometry imaging of rat brain sections: nanomolar sensitivity with MALDI versus nanometer resolution by TOF-SIMS. Anal Bioanal Chem 396(1):151-162. doi:10.1007/s00216-009-3031-2

Bruinen AL, van Oevelen C, Eijkel GB, Van Heerden M, Cuyckens F, Heeren RM (2016) Mass spectrometry imaging of drug related crystal-like structures in formalin-fixed frozen and paraffin-embedded rabbit kidney tissue sections. J Am Soc Mass Spectrom 27(1):117-123. doi:10.1007/s13361-015-1254-3

Burnum KE, Cornett DS, Puolitaival SM et al (2009) Spatial and temporal alterations of phospholipids determined by mass spectrometry during mouse embryo implantation. J Lipid Res 50(11):2290-2298. doi:10.1194/jlr.M900100-JLR200

Caprioli RM, Farmer TB, Gile J (1997) Molecular imaging of biological samples: localization of peptides and proteins using MALDITOF MS. Anal Chem 69(23):4751-4760

Castellino S, Groseclose MR, Wagner D (2011) MALDI imaging mass spectrometry: bridging biology and chemistry in drug development. Bioanalysis 3(21):2427-2441. doi:10.4155/ bio.11.232

Chumbley CW, Reyzer ML, Allen JL et al (2016) Absolute quantitative MALDI imaging mass spectrometry: a case of Rifampicin in liver tissues. Anal Chem 88(4):2392-2398. doi:10.1021/acs. analchem.5b04409

Colliver TL, Brummel CL, Pacholski ML, Swanek FD, Ewing AG, Winograd N (1997) Atomic and molecular imaging at the singlecell level with TOF-SIMS. Anal Chem 69(13):2225-2231

Cornett DS, Reyzer ML, Chaurand P, Caprioli RM (2007) MALDI imaging mass spectrometry: molecular snapshots of biochemical systems. Nat Methods 4(10):828-833. doi:10.1038/nmeth1094

Cox PA, Davis DA, Mash DC, Metcalf JS, Banack SA (2016) Dietary exposure to an environmental toxin triggers neurofibrillary tangles and amyloid deposits in the brain. Proc Biol Sci. doi:10.1098/rspb.2015.2397

Deininger SO, Ebert MP, Futterer A, Gerhard M, Rocken C (2008) MALDI imaging combined with hierarchical clustering as a new tool for the interpretation of complex human cancers. J Proteome Res 7(12):5230-5236. doi:10.1021/pr8005777

Drexler DM, Garrett TJ, Cantone JL et al (2007) Utility of imaging mass spectrometry (IMS) by matrix-assisted laser desorption ionization (MALDI) on an ion trap mass spectrometer in the analysis of drugs and metabolites in biological tissues. $\mathrm{J}$ Pharmacol Toxicol Methods 55(3):279-288. doi:10.1016/j. vascn.2006.11.004

Fisher GL, Bruinen AL, Ogrinc Potocnik N et al (2016) A new method and mass spectrometer design for TOF-SIMS parallel imaging MS/MS. Anal Chem 88(12):6433-6440. doi:10.1021/ acs.analchem.6b01022

Goodwin RJ, Dungworth JC, Cobb SR, Pitt AR (2008) Time-dependent evolution of tissue markers by MALDI-MS imaging. Proteomics 8(18):3801-3808. doi:10.1002/pmic.200800201

Graham DJ, Castner DG (2012) Multivariate analysis of ToF-SIMS data from multicomponent systems: the why, when, and how. Biointerphases 7(1-4):49. doi:10.1007/s13758-012-0049-3

Groseclose MR, Castellino S (2013) A mimetic tissue model for the quantification of drug distributions by MALDI imaging mass spectrometry. Anal Chem 85(21):10099-10106. doi:10.1021/ ac $400892 z$

Groseclose MR, Laffan SB, Frazier KS, Hughes-Earle A, Castellino S (2015) Imaging MS in toxicology: an investigation of juvenile rat nephrotoxicity associated with dabrafenib administration. J Am Soc Mass Spectrom 26(6):887-898. doi:10.1007/s13361-015-1103-4

Gustafsson OJ, Briggs MT, Condina MR et al (2015) MALDI imaging mass spectrometry of N-linked glycans on formalinfixed paraffin-embedded murine kidney. Anal Bioanal Chem 407(8):2127-2139. doi:10.1007/s00216-014-8293-7

Hamm G, Bonnel D, Legouffe R et al (2012) Quantitative mass spectrometry imaging of propranolol and olanzapine using tissue extinction calculation as normalization factor. J Proteomics 75(16):4952-4961. doi:10.1016/j.jprot.2012.07.035

Hanrieder J, Ljungdahl A, Falth M, Mammo SE, Bergquist J, Andersson M (2011) L-DOPA-induced dyskinesia is associated with regional increase of striatal dynorphin peptides as elucidated by 
imaging mass spectrometry. Mol Cell Proteomics 10(10):M111 00930. doi:10.1074/mcp.M111.009308

Hanrieder J, Ljungdahl A, Andersson M (2012) MALDI imaging mass spectrometry of neuropeptides in Parkinson's disease. J Vis Exp. doi:10.3791/3445

Hanrieder J, Ekegren T, Andersson M, Bergquist J (2013) MALDI imaging of post-mortem human spinal cord in amyotrophic lateral sclerosis. J Neurochem 124(5):695-707. doi:10.1111/ jnc. 12019

Hanrieder J, Gerber L, Persson Sandelius A, Brittebo EB, Ewing AG, Karlsson O (2014a) High resolution metabolite imaging in the hippocampus following neonatal exposure to the environmental toxin BMAA using ToF-SIMS. ACS Chem Neurosci 5(7):568 575. doi:10.1021/cn500039b

Hanrieder J, Karlsson O, Brittebo EB, Malmberg P, Ewing AG (2014b) Probing the lipid chemistry of neurotoxin-induced hippocampal lesions using multimodal imaging mass spectrometry. Surf Interface Anal 46(S1):375-378. doi:10.1002/sia.5418

Hanrieder J, Malmberg P, Ewing AG (2015) Spatial neuroproteomics using imaging mass spectrometry. Biochim Biophys Acta 185(7):718-731. doi:10.1016/j.bbapap.2014.12.026

Henderson A, Fletcher JS, Vickerman JC (2009) A comparison of PCA and MAF for ToF-SIMS image interpretation. Surf Interface Anal 41(8):666-674. doi:10.1002/sia.3084

Hillenkamp F, Karas M, Beavis RC, Chait BT (1991) Matrix-assisted laser desorption/ionization mass spectrometry of biopolymers. Anal Chem 63(24):1193A-1203A

Hochart G, Hamm G, Stauber J (2014) Label-free MS imaging from drug discovery to preclinical development. Bioanalysis 6(20):2775-2788. doi:10.4155/bio.14.202

Jones EA, van Remoortere A, van Zeijl RJ et al (2011) Multiple statistical analysis techniques corroborate intratumor heterogeneity in imaging mass spectrometry datasets of myxofibrosarcoma. PLoS ONE 6(9):e24913. doi:10.1371/journal.pone.0024913

Jurchen JC, Rubakhin SS, Sweedler JV (2005) MALDI-MS imaging of features smaller than the size of the laser beam. J Am Soc Mass Spectrom 16(10):1654-1659. doi:10.1016/j. jasms.2005.06.006

Kadar H, Le Douaron G, Amar M et al (2014) MALDI mass spectrometry imaging of 1-methyl-4-phenylpyridinium (MPP+) in mouse brain. Neurotox Res 25(1):135-145. doi:10.1007/ s12640-013-9449-5

Kallback P, Shariatgorji M, Nilsson A, Andren PE (2012) Novel mass spectrometry imaging software assisting labeled normalization and quantitation of drugs and neuropeptides directly in tissue sections. J Proteomics 75(16):4941-4951. doi:10.1016/j. jprot.2012.07.034

Karas M, Hillenkamp F (1988) Laser desorption ionization of proteins with molecular masses exceeding 10,000 daltons. Anal Chem 60(20):2299-2301

Karlsson O, Lindquist NG (2016) Melanin and neuromelanin binding of drugs and chemicals: toxicological implications. Arch Toxicol 90(8):1883-1891. doi:10.1007/s00204-016-1757-0

Karlsson O, Lindquist NG, Brittebo EB, Roman E (2009a) Selective brain uptake and behavioral effects of the cyanobacterial toxin BMAA (beta- $N$-methylamino-L-alanine) following neonatal administration to rodents. Toxicol Sci 109(2):286-295. doi:10.1093/toxsci/kfp062

Karlsson O, Roman E, Brittebo EB (2009b) Long-term cognitive impairments in adult rats treated neonatally with beta- $N$-Methylamino-L-Alanine. Toxicol Sci 112(1):185-195. doi:10.1093/ toxsci/kfp196

Karlsson O, Roman E, Berg AL, Brittebo EB (2011) Early hippocampal cell death, and late learning and memory deficits in rats exposed to the environmental toxin BMAA (beta- $N$-methylamino-L-alanine) during the neonatal period. Behav Brain Res 219(2):310-320. doi:10.1016/j.bbr.2011.01.056

Karlsson O, Berg AL, Lindstrom AK et al (2012) Neonatal exposure to the cyanobacterial toxin BMAA induces changes in protein expression and neurodegeneration in adult hippocampus. Toxicol Sci 130(2):391-404. doi:10.1093/toxsci/kfs241

Karlsson O, Kultima K, Wadensten H et al (2013) Neurotoxininduced neuropeptide perturbations in striatum of neonatal rats. $\mathrm{J}$ Proteome Res 12(4):1678-1690. doi:10.1021/pr3010265

Karlsson O, Bergquist J, Andersson M (2014) Quality measures of imaging mass spectrometry aids in revealing long-term striatal protein changes induced by neonatal exposure to the cyanobacterial toxin beta- $N$-methylamino-L-alanine (BMAA). Mol Cell Proteomics 13(1):93-104. doi:10.1074/mcp.M113.031435

Karlsson O, Berg AL, Hanrieder J, Arnerup G, Lindstrom AK, Brittebo EB (2015a) Intracellular fibril formation, calcification, and enrichment of chaperones, cytoskeletal, and intermediate filament proteins in the adult hippocampus CA1 following neonatal exposure to the nonprotein amino acid BMAA. Arch Toxicol 89(3):423-436. doi:10.1007/s00204-014-1262-2

Karlsson O, Jiang L, Ersson L, Malmstrom T, Ilag LL, Brittebo EB (2015b) Environmental neurotoxin interaction with proteins: dose-dependent increase of free and protein-associated BMAA (beta- $N$-methylamino-L-alanine) in neonatal rat brain. Sci Rep 5:15570. doi:10.1038/srep15570

Khatib-Shahidi S, Andersson M, Herman JL, Gillespie TA, Caprioli RM (2006) Direct molecular analysis of whole-body animal tissue sections by imaging MALDI mass spectrometry. Anal Chem 78(18):6448-6456. doi:10.1021/ac060788p

Koeniger SL, Talaty N, Luo Y et al (2011) A quantitation method for mass spectrometry imaging. Rapid Commun Mass Spectrom 25(4):503-510. doi:10.1002/rcm.4891

Kriegsmann M, Seeley EH, Schwarting A et al (2012) MALDI MS imaging as a powerful tool for investigating synovial tissue. Scand J Rheumatol 41(4):305-309. doi:10.3109/03009742.201 1.647925

Lagarrigue M, Lavigne R, Tabet E et al (2014) Localization and in situ absolute quantification of chlordecone in the mouse liver by MALDI imaging. Anal Chem 86(12):5775-5783. doi:10.1021/ac500313s

Lanao JM, Fraile MA (2005) Drug tissue distribution: study methods and therapeutic implications. Curr Pharm Des 11(29):3829-3845

Laskin J, Heath BS, Roach PJ, Cazares L, Semmes OJ (2012) Tissue imaging using nanospray desorption electrospray ionization mass spectrometry. Anal Chem 84(1):141-148. doi:10.1021/ ac2021322

Lemaire R, Desmons A, Tabet JC, Day R, Salzet M, Fournier I (2007) Direct analysis and MALDI imaging of formalin-fixed, paraffin-embedded tissue sections. J Proteome Res 6(4):1295-1305. doi:10.1021/pr060549i

Ljungdahl A, Hanrieder J, Falth M, Bergquist J, Andersson M (2011) Imaging mass spectrometry reveals elevated nigral levels of dynorphin neuropeptides in L-DOPA-induced dyskinesia in rat model of Parkinson's disease. PLoS ONE 6(9):e25653. doi:10.1371/journal.pone.0025653

Ljungdahl A, Hanrieder J, Bergquist J, Andersson M (2013) Analysis of neuropeptides by MALDI imaging mass spectrometry. Methods Mol Biol 1023:121-136. doi:10.1007/978-1-4614-7209-4_7

Ly A, Buck A, Balluff B et al (2016) High-mass-resolution MALDI mass spectrometry imaging of metabolites from formalin-fixed paraffin-embedded tissue. Nat Protoc 11(8):1428-1443. doi:10.1038/ nprot.2016.081

Martin-Lorenzo M, Balluff B, Sanz-Maroto A et al (2014) $30 \mu \mathrm{m}$ spatial resolution protein MALDI MSI: in-depth comparison of five sample preparation protocols applied to human 
healthy and atherosclerotic arteries. J Proteomics 108:465-468. doi:10.1016/j.jprot.2014.06.013

McDonnell LA, Heeren RM (2007) Imaging mass spectrometry. Mass Spectrom Rev 26(4):606-643. doi:10.1002/mas.20124

McEwen A, Henson C (2015) Quantitative whole-body autoradiography: past, present and future. Bioanalysis 7(5):557-568. doi:10.4155/bio.15.9

McEwen AB, Henson CM, Wood SG (2014) Quantitative whole-body autoradiography, LC-MS/MS and MALDI for drug-distribution studies in biological samples: the ultimate matrix trilogy. Bioanalysis 6(3):377-391. doi:10.4155/bio.13.336

Meistermann H, Norris JL, Aerni HR et al (2006) Biomarker discovery by imaging mass spectrometry: transthyretin is a biomarker for gentamicin-induced nephrotoxicity in rat. Mol Cell Proteomics 5(10):1876-1886. doi:10.1074/mcp.M500399-MCP200

Mouton JW, Theuretzbacher U, Craig WA, Tulkens PM, Derendorf H, Cars O (2008) Tissue concentrations: do we ever learn? J Antimicrob Chemother 61(2):235-237. doi:10.1093/jac/dkm476

Nilsson A, Falth M, Zhang X et al (2009) Striatal alterations of secretogranin-1, somatostatin, prodynorphin, and cholecystokinin peptides in an experimental mouse model of Parkinson disease. Mol Cell Proteomics 8(5):1094-1104. doi:10.1074/mcp. M800454-MCP200

Nilsson A, Fehniger TE, Gustavsson L et al (2010) Fine mapping the spatial distribution and concentration of unlabeled drugs within tissue micro-compartments using imaging mass spectrometry. PLoS ONE 5(7):e11411. doi:10.1371/journal.pone.0011411

Nilsson A, Forngren B, Bjurstrom S et al (2012) In situ mass spectrometry imaging and ex vivo characterization of renal crystalline deposits induced in multiple preclinical drug toxicology studies. PLoS ONE 7(10):e47353. doi:10.1371/journal. pone. 0047353

Nilsson A, Goodwin RJ, Swales JG et al (2015) Investigating nephrotoxicity of polymyxin derivatives by mapping renal distribution using mass spectrometry imaging. Chem Res Toxicol 28(9):1823-1830. doi:10.1021/acs.chemrestox.5b00262

Norris JL, Cornett DS, Mobley JA et al (2007) Processing MALDI mass spectra to improve mass spectral direct tissue analysis. Int $\mathbf{J}$ Mass Spectrom 260(2-3):212-221

Northen TR, Yanes O, Northen MT et al (2007) Clathrate nanostructures for mass spectrometry. Nature 449(7165):1033-1036. doi:10.1038/nature06195

Ogrinc Potocnik N, Porta T, Becker M, Heeren RM, Ellis SR (2015) Use of advantageous, volatile matrices enabled by next-generation high-speed matrix-assisted laser desorption/ionization time-of-flight imaging employing a scanning laser beam. Rapid Commun Mass Spectrom 29(23):2195-2203. doi:10.1002/ rcm.7379

Onishi S, Tatsumi Y, Wada K et al (2013) Sulfatide accumulation in the dystrophic terminals of gracile axonal dystrophy mice: lipid analysis using matrix-assisted laser desorption/ionization imaging mass spectrometry. Med Mol Morphol. doi:10.1007/ s00795-013-0019-y

Oppenheimer SR, Mi D, Sanders ME, Caprioli RM (2010) Molecular analysis of tumor margins by MALDI mass spectrometry in renal carcinoma. J Proteome Res 9(5):2182-2190. doi:10.1021/ pr900936z

Pellegatti M, Pagliarusco S (2011) Drug and metabolite concentrations in tissues in relationship to tissue adverse findings: a review. Expert Opin Drug Metab Toxicol 7(2):137-146. doi:10.1517/1742 5255.2011.545053

Pierson J, Norris JL, Aerni HR, Svenningsson P, Caprioli RM, Andren PE (2004) Molecular profiling of experimental Parkinson's disease: direct analysis of peptides and proteins on brain tissue sections by MALDI mass spectrometry. J Proteome Res 3(2):289-295
Prentice BM, Chumbley CW, Caprioli RM (2016) Absolute quantification of rifampicin by MALDI Imaging mass spectrometry using multiple TOF/TOF events in a single laser shot. J Am Soc Mass Spectrom. doi:10.1007/s13361-016-1501-2

Rohner TC, Staab D, Stoeckli M (2005) MALDI mass spectrometric imaging of biological tissue sections. Mech Ageing Dev 126(1):177-185. doi:10.1016/j.mad.2004.09.032

Schwamborn K, Caprioli RM (2010) MALDI imaging mass spectrometry-painting molecular pictures. Mol Oncol 4(6):529-538. doi:10.1016/j.molonc.2010.09.002

Seeley EH, Oppenheimer SR, Mi D, Chaurand P, Caprioli RM (2008) Enhancement of protein sensitivity for MALDI imaging mass spectrometry after chemical treatment of tissue sections. J Am Soc Mass Spectrom 19(8):1069-1077. doi:10.1016/j. jasms.2008.03.016

Shariatgorji M, Kallback P, Gustavsson L et al (2012a) Controlled$\mathrm{pH}$ tissue cleanup protocol for signal enhancement of small molecule drugs analyzed by MALDI-MS imaging. Anal Chem 84(10):4603-4607. doi:10.1021/ac203322q

Shariatgorji M, Nilsson A, Goodwin RJA et al (2012b) Deuterated matrix-assisted laser desorption ionization matrix uncovers masked mass spectrometry imaging signals of small molecules. Anal Chem 84(16):7152-7157. doi:10.1021/ac301498m

Shariatgorji M, Nilsson A, Kallback P et al (2015) Pyrylium salts as reactive matrices for MALDI-MS imaging of biologically active primary amines. J Am Soc Mass Spectrom 26(6):934-939. doi:10.1007/s13361-015-1119-9

Skold K, Svensson M, Nilsson A et al (2006) Decreased striatal levels of PEP-19 following MPTP lesion in the mouse. J Proteome Res 5(2):262-269. doi:10.1021/pr050281f

Solon EG, Schweitzer A, Stoeckli M, Prideaux B (2010) Autoradiography, MALDI-MS, and SIMS-MS imaging in pharmaceutical discovery and development. AAPS J 12(1):11-26. doi:10.1208/ s12248-009-9158-4

Spencer PS, Nunn PB, Hugon J et al (1987) Guam amyotrophic lateral sclerosis-parkinsonism-dementia linked to a plant excitant neurotoxin. Science 237(4814):517-522

Spengler B, Hubert M (2002) Scanning microprobe matrix-assisted laser desorption ionization (SMALDI) mass spectrometry: instrumentation for sub-micrometer resolved LDI and MALDI surface analysis. J Am Soc Mass Spectrom 13(6):735-748. doi:10.1016/S1044-0305(02)00376-8

Stauber J, Lemaire R, Franck J et al (2008) MALDI imaging of formalin-fixed paraffin-embedded tissues: application to model animals of Parkinson disease for biomarker hunting. J Proteome Res 7(3):969-978. doi:10.1021/pr070464x

Stoeckli M, Staab D, Schweitzer A (2007) Compound and metabolite distribution measured by MALDI mass spectrometric imaging in whole-body tissue sections. Int J Mass Spectrom 260(2-3):195202. doi:10.1016/j.ijms.2006.10.007

Sugiura Y, Setou M (2010) Imaging mass spectrometry for visualization of drug and endogenous metabolite distribution: toward in situ pharmacometabolomes. J Neuroimmune Pharmacol 5(1):31-43. doi:10.1007/s11481-009-9162-6

Takai N, Tanaka Y, Watanabe A, Saji H (2013) Quantitative imaging of a therapeutic peptide in biological tissue sections by MALDI MS. Bioanalysis 5(5):603-612. doi:10.4155/bio.13.13

Takats Z, Wiseman JM, Gologan B, Cooks RG (2004) Mass spectrometry sampling under ambient conditions with desorption electrospray ionization. Science 306(5695):471-473. doi:10.1126/science.1104404

Tyler BJ, Rayal G, Castner DG (2007) Multivariate analysis strategies for processing ToF-SIMS images of biomaterials. Biomaterials 28(15):2412-2423. doi:10.1016/j.biomaterials.2007.02.002

Ullberg S (1954) Studies on the distribution and fate of ${ }^{35}$ S-labelled benzylpenicillin in the body. Acta Radiol Suppl 118:1-110 
Ullberg S (1977) The technique of whole body autroradiography. Cryosectioning of large specimens. Sci Tools LKB Instr J, Special issue: $1-29$

Ungerstedt U (1968) 6-Hydroxy-dopamine induced degeneration of central monoamine neurons. Eur J Pharmacol 5(1):107

Uys JD, Grey AC, Wiggins A, Schwacke JH, Schey KL, Kalivas PW (2010) Matrix-assisted laser desorption/ionization tissue profiling of secretoneurin in the nucleus accumbens shell from cocainesensitized rats. J Mass Spectrom 45(1):97-103. doi:10.1002/ jms. 1697

Waki M, Sugiyama E, Kondo T, Sano K, Setou M (2015) Nanoparticle-assisted laser desorption/ionization for metabolite imaging. Methods Mol Biol 1203:159-173. doi:10.1007/978-1-4939-1357-2_16

Willems SM, van Remoortere A, van Zeijl R, Deelder AM, McDonnell LA, Hogendoorn PC (2010) Imaging mass spectrometry of myxoid sarcomas identifies proteins and lipids specific to tumour type and grade, and reveals biochemical intratumour heterogeneity. $\mathbf{J}$ Pathol 222(4):400-409. doi:10.1002/path.2771

Yanagisawa K, Shyr Y, Xu BJ et al (2003) Proteomic patterns of tumour subsets in non-small-cell lung cancer. Lancet 362(9382):433-439. doi:10.1016/S0140-6736(03)14068-8 